\title{
ON UNIFORM DISTRIBUTION OF ALGEBRAIC NUMBERS
}

L. G. PECK

Let $K$ be an algebraic number field of degree $n+1$ over the rational field $R$. The conjugates of $K$ are denoted by $K^{(0)}, \cdots, K^{(n)}$, it being supposed that $K^{(0)}, \cdots, K^{\left(r_{1}\right)}$ are real, $K^{\left(r_{1}+1\right)}, \cdots, K^{(n)}$ are complex, and moreover that for $k=r_{1}+1, \cdots, r_{1}+r_{2}$ the fields $K^{(k)}$ and $K^{\left(k+r_{2}\right)}$ are obtainable from one another by interchanging $i\left(=(-1)^{1 / 2}\right)$ and $-i$ (here $r_{1}+2 r_{2}=n$ ). I assume throughout that $r_{1} \geqq 0$ and that $n \geqq 1$. Numbers in $K$ are represented by small Greek letters, the insertion of superscripts denoting the passage to the corresponding conjugates. Let $\omega_{0}, \cdots, \omega_{n}$ be a set of numbers in $K$ which are linearly independent over $R$; let $\omega_{0}=1$; and finally let $f\left(x_{1}, \cdots, x_{n}\right)$ be a (complex-valued) function of $n$ real variables which is Riemann integrable over the unit cube $E_{n}: 0<x_{k}<1 \quad(k=1, \cdots, n)$. Then, according to theorems of Weyl on uniform distribution,

$$
\begin{aligned}
\int_{E_{n}} f\left(x_{1}, \cdots, x_{n}\right) d x_{1} & \cdots d x_{n} \\
= & \frac{1}{M} \sum_{m=0}^{M-1} f\left(\left\{m \omega_{1}^{(0)}\right\}, \cdots,\left\{m \omega_{n}^{(0)}\right\}\right)+o(1)
\end{aligned}
$$

as $M \rightarrow \infty$, the braces denoting the fractional part. The object of this paper is to show that the error term in (1) may be replaced by $O(1 / M)$ provided $f$ satisfies certain additional conditions.

More precisely, suppose that in $E_{n}$

$$
f\left(x_{1}, \cdots, x_{n}\right)=\sum_{q_{1}, \cdots, q_{n}=-\infty}^{\infty} a\left(q_{1}, \cdots, q_{n}\right) e^{2 i \pi\left(q_{1} x_{1}+\cdots+q_{n} x_{n}\right)},
$$

the series on the right being absolutely convergent.

TheOREM. If there exist positive constants $C$ and $c$ such that

$$
\left|a\left(q_{1}, \cdots, q_{n}\right)\right|<C\left(\left|q_{1}\right|+\cdots+\left|q_{n}\right|\right)^{-n-c} \text {, }
$$

then the expression

$$
S_{M}=M \int_{E_{n}} f\left(x_{1}, \cdots, x_{n}\right) d x_{1} \cdots d x_{n}
$$

$$
-\sum_{m=0}^{M-1} f\left(m \omega_{1}^{(0)}, \cdots, m \omega_{n}^{(0)}\right)
$$

Received by the editors December 11, 1951. 
is bounded in absolute value with a bound which depends only on $C, c, \omega_{1}$, $\cdots, \omega_{n}$ (and not on $M$ ).

The theorem probably remains true when (3) is replaced by the weaker condition

$$
\left|a\left(q_{1}, \cdots, q_{n}\right)\right|<C\left(1+\left|q_{1}\right|\right)^{-1-c} \cdots\left(1+\left|q_{n}\right|\right)^{-1-c},
$$

but I have been unable to prove this.

For the proof of the theorem, it is clear from (2) and (4) that

$$
\begin{aligned}
& S_{M}=\sum_{q_{1}, \cdots, q_{n}}^{\prime} a\left(q_{1}, \cdots, q_{n}\right) \frac{\sin \pi M\left(q_{1} \omega_{1}^{(0)}+\cdots+q_{n} \omega_{n}^{(0)}\right)}{\sin \pi\left(q_{1} \omega_{1}^{(0)}+\cdots+q_{n} \omega_{n}^{(0)}\right)} \\
& \cdot e^{i \pi(M-1)\left(q_{1} \omega_{1}^{0}+\cdots+q_{n} \omega_{n}^{(0)}\right)}
\end{aligned}
$$

where the ' indicates that $q_{1}=\cdots=q_{n}=0$ is to be omitted in summation. Thus, from (3),

$$
\left|S_{M}\right|<\frac{C}{2} \sum^{\prime}\left(\left|q_{1}\right|+\cdots+\left|q_{n}\right|\right)^{-n-c}
$$

$$
\cdot\left\|q_{1} \omega_{1}^{(0)}+\cdots+q_{n} \omega_{n}^{(0)}\right\|^{-1}
$$

where $\|x\|$ stands for $\min _{k=0, \pm 1, \pm 2}, \ldots|x-k|$.

Let $\mathfrak{P}$ denote the set of numbers $\alpha$ in $K$ of the form

$$
\alpha=q_{0}+q_{1} \omega_{1}+\cdots+q_{n} \omega_{n}
$$

with rational integers $q_{0}, \cdots, q_{n}$. An element $\alpha$ of $\mathfrak{A}$ is uniquely determined by the numbers $q_{1}, \cdots, q_{n}$ and the condition

$$
\left|\alpha^{(0)}\right|<1 / 2 \text {. }
$$

When this condition is satisfied, one sees that

$$
\left|q_{0}\right|<\max _{k=1, \cdots, n}\left|\omega_{k}^{(0)}\right| \cdot\left(\left|q_{1}\right|+\cdots+\left|q_{n}\right|\right)+1 / 2
$$

i.e.,

$$
\left|q_{0}\right|<C_{1}\left(\left|q_{1}\right|+\cdots+\left|q_{n}\right|\right),
$$

whence

$$
\left|\alpha^{(k)}\right|<C_{2}\left(\left|q_{1}\right|+\cdots+\left|q_{n}\right|\right) \quad(k=1, \cdots, n)
$$

with

$$
C_{2}=1 / 2+\max _{k=1, \cdots, n}\left|\omega_{k}^{(0)}\right|+\max _{j, k=1, \cdots, n}\left|\omega_{k}^{(j)}\right|
$$


Thus,

$$
\left|S_{M}\right|<C_{3} \sum_{\alpha \text { in } \mathfrak{x}, 0>\left|\alpha^{(0)}\right|<1 / 2}|N(\alpha)|^{-1}\left(\max _{k=1, \cdots, n}\left|\alpha^{(k)}\right|\right)^{-c} .
$$

The ideal $\left(1, \omega_{1}, \cdots, \omega_{n}\right)$ may be written $\mathfrak{a} / m$ where $\mathfrak{a}$ is an integral ideal and $m$ is a positive rational integer. The elements of $\mathfrak{A}$ are, of course, all divisible by $a / m$; hence

$$
\left|S_{M}\right|<C_{4} \sum_{a|\alpha, 0<| \alpha^{(0)} \mid<m / 2}|N(\alpha)|^{-1}\left(\max _{k=1, \cdots, n}\left|\alpha^{(k)}\right|\right)^{-c} .
$$

It is, therefore, sufficient to prove that the sum on the right side of (5), with $\mathfrak{a}=(1)$, is convergent.

Let $\mathbf{P}$ be a complete set of nonassociated integers of $K$, so that every integer of $K$ is uniqueily expressible as the product of a unit by an element of $P$. Then the sum in (5) with $a=(1)$ may be written

$$
S=\sum_{\alpha \text { in } P}|N(\alpha)|^{-1} \sum_{|N(\epsilon)|=1,0<\left|\epsilon^{(0)} \alpha^{(0)}\right|<m / 2}\left(\max _{k=1, \cdots, n}\left|\epsilon^{(k)} \alpha^{(k)}\right|\right)^{-c},
$$

where $\epsilon$, in the inner sum, runs over all units satisfying the indicated condition. If the elements $\alpha$ of $\mathbf{P}$ are chosen so that

$$
C_{5}|N(\alpha)|^{1 / n}>\alpha^{(k)}>|N(\alpha)|^{1 / n} \quad(k=1, \cdots, n)
$$

(which according to the Dirichlet theory of units is always possible for a constant $C_{5}$ which depends only on the regulator of $K$ ), then

$$
\begin{aligned}
S & \leqq \sum_{\alpha \text { in } P}|N(\alpha)|^{-1-c / n} \sum_{|N(\epsilon)|=1,0<\left|\epsilon^{(0)} \alpha^{(0)}\right|<m / 2}\left(\max _{k=1, \cdots, n}\left|\epsilon^{(k)}\right|\right)^{-c} \\
& \leqq \zeta_{K}\left(1+\frac{c}{n}\right) \sum_{|N(\epsilon)|=1,\left|\epsilon^{(0)}\right|<C_{b}}\left(\max _{k=1, \cdots, n}\left|\epsilon^{(k)}\right|\right)^{-c}
\end{aligned}
$$

where $\zeta_{K}$ is the Dirichlet zeta function for the field $K$ and $C_{6}=m C_{5}^{n} / 2$ is a constant which depends only on the numbers $\omega_{1}, \cdots, \omega_{n}$ and on the regulator of $K$.

Appealing once more to the Dirichlet theory of units, one sees from the $r\left(=r_{1}+r_{2}\right)$-dimensional lattice formed by the logarithms of the units that there is a constant $C_{7}>0$ such that at most one unit $\epsilon$ (aside from multiplicative factors of roots of unity) satisfies

$$
t_{k} \leqq \log \left|\epsilon^{(k)}\right|<t_{k}+C_{7} \quad(k=1, \cdots, n),
$$

the real numbers $t_{1}, \cdots, t_{n}$ being arbitrarily prescribed in advance with the convention that $t_{k+r_{3}}=t_{k}$ for $k=r_{1}+1, \cdots, r_{1}+r_{2}$. Note that 


$$
-n C_{7}-\left(t_{1}+\cdots+t_{n}\right)<\log \left|\epsilon^{(0)}\right| \leqq-\left(t_{1}+\cdots+t_{n}\right)
$$

is a consequence of (7).

Since $r_{1} \geqq 0$, the only roots of unity in $K$ are \pm 1 ; therefore, choosing

$$
t_{k}=m_{k} C_{7}
$$$$
(k=1, \cdots, r)
$$

where $m_{1}, \cdots, m_{r}$ are rational integers, one deduces from (6), (7), (8) that

$$
S<2 \zeta_{k}\left(1+\frac{c}{n}\right) \sum e^{-c C_{7} \max \left(m_{1}, \cdots, m_{r}\right)}
$$

where the sum runs over all sets of rational integers $m_{1}, \cdots, m_{r}$ such that $-\left(m_{1}+\cdots+m_{r_{1}}\right)-2\left(m_{r_{1}+1}+\cdots+m_{r_{1}+r_{2}}\right)<\log C_{6} / C_{7}=-C_{8}$, say. Thus

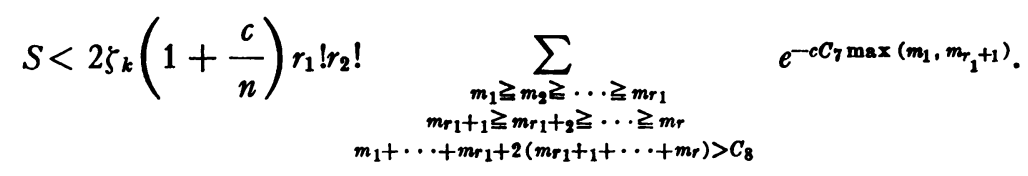

The latter sum may be divided into two sections in the first of which $m_{1} \geqq m_{r_{1}+1}$ and in the second of which $m_{r_{1}+1}>m_{1}$.

In the first section the inequalities under the summation sign imply $m_{r_{1}}>C_{8}-(n-1) m_{1}$ and $m_{r}>\left(C_{8}-(n-2) m_{1}\right) / 2$ so that the number of summands corresponding to a particular $m_{1}$ is at most $\left(n m_{1}-C_{8}\right)^{r-1} \cdot 2^{-r_{2}}$ or 0 according as $m_{1}>C_{8} / n$ or $m_{1} \leqq C_{8} / n$. In the second section of the sum, the inequalities imply $m_{r_{1}}>C_{8}-(n-1) m_{r_{1}+1}$ and $m_{r}>\left(C_{8}-(n-2) m_{r_{1}+1}\right) / 2$, so that the number of summands corresponding to a particular $m_{r_{1}+1}$ is at most $\left(n m_{r_{1}+1}-C_{8}\right)^{r_{-1}} \cdot 2^{-r_{2}+1}$ or 0 according as $m_{r_{1}+1}>C_{8} / n$ or $m_{r_{1}+1} \leqq C_{8} / n$. Thus,

(9) $S<3 \cdot 2^{-r_{2}+1} r_{1} ! r_{2} ! \zeta_{K}\left(1+\frac{c}{n}\right) \sum_{m=1+\left[C_{8} / n\right]}^{\infty}\left(n m-C_{8}\right)^{r^{-1}} e^{-m c C_{7}}$

which is clearly finite.

It is interesting to observe, from (9), that the bound for $S$ is $O\left(c^{-r-1}\right)$.

Los Alamos Scientific Laboratory 